Amin Farjudian, ${ }^{*}$ School of Computer Science, The University of Nottingham Ningbo China, 199 Taikang East Road, Ningbo, 315100, China. email: Amin.Farjudian@nottingham.edu.cn

Behrouz Emamizadeh, School of Mathematical Sciences, The University of Nottingham Ningbo China, 199 Taikang East Road, Ningbo, 315100, China. email: Behrouz.Emamizadeh@nottingham.edu.cn

\title{
ABSOLUTE CONTINUITY IN PARTIAL DIFFERENTIAL EQUATIONS
}

\begin{abstract}
In this note we study a function which frequently appears in partial differential equations. We prove that this function is absolutely continuous, hence it can be written as a definite integral. As a result we obtain some estimates regarding solutions of the Hamilton-Jacobi systems.
\end{abstract}

\section{Introduction}

Let $H$ be a differential operator of order $m \in \mathbb{N}$ and let $f \in L^{p}(D)$ be a positive function, where $p \in(1, \infty)$ and $D$ is a smooth bounded domain in $\mathbb{R}^{n}$. Consider the equation:

$$
H(u)=f, \quad \text { in } D
$$

A function $u \in W^{m, p}(D) \cap C(\bar{D})$ is called a strong solution of (1) provided that $H(u)=$ $f$ almost everywhere (a. e.) in $D$. We assume the operator $H$ satisfies the following condition:

For any $u \in W^{m}(D)$ and $\gamma \in \mathbb{R}: H(u)=0$ a.e. in $E_{\gamma}:=\{x \in D \mid u(x)=\gamma\}$

\footnotetext{
Mathematical Reviews subject classification: Primary: 28D05, 35F2

Key words: Absolute continuity, Weak convergence, Rearrangements of functions, Measure preserving maps, Hamilton-Jacobi systems

*Amin Farjudian's work on this article has been partially supported by the Natural Science Foundation of China (Grant No. 61070023) and Ningbo Natural Science Programme by Ningbo S\&T bureau (Grant No. 2010A610104).
} 
For a measurable function $h: D \rightarrow \mathbb{R}$, the distribution function of $h$, denoted $\lambda_{h}(\alpha)$, is defined as follows:

$$
\lambda_{h}(\alpha):=|\{x \in D \mid h(x) \geq \alpha\}| \equiv|\{h \geq \alpha\}|, \quad(\forall \alpha \in \mathbb{R})
$$

where $|\cdot|$ denotes the $n$-dimensional Lebesgue measure. Clearly $\lambda_{h}$ is decreasing, and if $h$ is continuous, then $\lambda_{h}$ will be strictly decreasing. Moreover, in case the graph of $h$ has no significant flat sections (i.e. $\forall \gamma \in \mathbb{R}:|\{h=\gamma\}|=0$ ) then $\lambda_{h}$ will be continuous. The decreasing rearrangement of $h$, denoted $h^{*}(s)$, is defined as follows:

$$
\left\{\begin{array}{l}
h^{*}:[0,|D|] \rightarrow \mathbb{R} \\
h^{*}(s)=\inf \left\{\alpha \mid \lambda_{h}(\alpha) \leq s\right\}
\end{array}\right.
$$

Note that when $h$ is continuous and its graph has no significant flat sections then:

$$
\lambda_{h} \circ h^{*}(s)=s \text { and } h^{*} \circ \lambda_{h}(\alpha)=\alpha .
$$

We also need to recall some background from rearrangements of functions. Given $g_{0}: D \subseteq \mathbb{R}^{n} \rightarrow \mathbb{R}$, the rearrangement class generated by $g_{0}$, denoted $\mathcal{R}\left(g_{0}\right)$, is the set of functions $g: D \rightarrow \mathbb{R}$ such that $\lambda_{g}(\alpha)=\lambda_{g_{0}}(\alpha)$, for every real $\alpha$. In case $g_{0} \in L^{p}(D)$ then $\mathcal{R}\left(g_{0}\right) \subseteq L^{p}(D)$, and $\forall g \in \mathcal{R}\left(g_{0}\right):\|g\|_{p}=\left\|g_{0}\right\|_{p}$. The weak closure of $\mathcal{R}\left(g_{0}\right)$ in $L^{p}(D)$ is denoted as $\overline{\mathcal{R}\left(g_{0}\right)}$ which, unlike $\mathcal{R}\left(g_{0}\right)$, enjoys some nice properties and characterizations that are stated in the following lemma. For the proof and further reading see $[3,4,5,9]$ :

Lemma 1. Let $g_{0} \in L^{p}(D)$ be a non-negative function, and $\mathcal{R}\left(g_{0}\right)$ be the rearrangement class generated by $g_{0}$. Then:

(1) $\overline{\mathcal{R}\left(g_{0}\right)}$ is convex, and weakly compact in $L^{p}(D)$.

(2) $\overline{\mathcal{R}\left(g_{0}\right)}=\overline{\operatorname{co}\left(\mathcal{R}\left(g_{0}\right)\right)}$, the closed convex hull of $\mathcal{R}\left(g_{0}\right)$.

(3) The following characterization stands:

$$
\begin{aligned}
\overline{\mathcal{R}\left(g_{0}\right)}=\{g \mid \forall s \in(0,|D|): \\
\left.\qquad \int_{0}^{s} g^{*}(t) d t \leq \int_{0}^{s} g_{0}^{*}(t) d t, \text { and } \int_{0}^{|D|} g^{*}(t) d t=\int_{0}^{|D|} g_{0}^{*}(t) d t\right\}
\end{aligned}
$$

The set of measure-preserving maps from $D$ onto $[0,|D|]$ is a non-empty set (e.g. see [12, Chapter 11]) which will be denoted by $\mathcal{M}(D,[0,|D|])$. By a result 
attributed to Ryff [13], given $g: D \rightarrow \mathbb{R}$, there exists $\phi \in \mathcal{M}(D,[0,|D|])$ such that $g=g^{*} \circ \phi$ almost everywhere in $D$.

We now introduce the function that is the main drive behind writing this note. To this end, we assume $u \in W^{m, p}(D) \cap C(\bar{D})$ is a strong solution of (1). We are interested in the function $\xi:[0,|D|] \rightarrow \mathbb{R}$ defined by:

$$
\xi(s)=\int_{\left\{u \geq u^{*}(s)\right\}} f(x) d x .
$$

Thanks to property (P) on page 1, and of course the fact that $f$ is positive, the level sets $\{u=\gamma\}$ must have zero measure, hence $\xi$ is well-defined. This function is frequently referred to in partial differential equations, particularly when one is interested in comparing the solution of a boundary value problem to that of a symmetrized problem, the latter being readily solved. There are many references in this regard, e. g. $[2,6,14]$, to mention a few. In this note we prove that $\xi$ is absolutely continuous, hence it can be represented by a definite integral of the form $\int_{0}^{s} F(\tau) d \tau$. Then, we will prove that the integrand $F$ composed with any measure-preserving map $\phi \in \mathcal{M}(D,[0,|D|])$ belongs to $\overline{\mathcal{R}(f)}$. Using these two results we point out a couple of applications.

Throughout this paper we use some standard notations. For example, $W^{m, p}(D)$ and $W^{m}(D)$ denote the usual Sobolev spaces. The space $L^{p}(D)$ comprises functions whose $p$-th powers are integrable, and the norm in this space is defined by $\|f\|_{p}=$ $\left(\int_{D}|f|^{p} d x\right)^{1 / p}$. Moreover, $C(D)$ and $C(\bar{D})$ denote the spaces of continuous functions over $D$ and its closure $\bar{D}$, respectively, and the corresponding norm is denoted by $\|\cdot\|_{\infty}$. The arrow " $\rightarrow$ " indicates strong convergence, whilst " $\rightarrow$ " indicates weak convergence in spaces under discussion.

\section{Main results}

Our first main result is the following:

Theorem 2. The function $\xi$, as defined in (2), is absolutely continuous on $[0,|D|]$.

Proof. Let $\epsilon>0$, and consider a finite sequence $\left\{\left(\alpha_{i}, \beta_{i}\right) \mid 1 \leq i \leq N\right\}$ of nonoverlapping subintervals of $[0,|D|]$ such that $\sum_{i=1}^{N}\left(\beta_{i}-\alpha_{i}\right)<\delta$, where $\delta$ is a positive number to be determined later. By setting $t\left(\alpha_{i}\right)=u^{*}\left(\alpha_{i}\right)$ and $t\left(\beta_{i}\right)=u^{*}\left(\beta_{i}\right)$ we will have:

$$
\sum_{i=1}^{N}\left|\xi\left(\beta_{i}\right)-\xi\left(\alpha_{i}\right)\right|=\sum_{i=1}^{N}\left|\int_{\left\{t\left(\beta_{i}\right)<u<t\left(\alpha_{i}\right)\right\}} f(x) d x\right|=\int_{E} f(x) d x
$$


where $E=\cup_{i=1}^{N}\left\{x: u^{*}\left(\beta_{i}\right)<u(x)<u^{*}\left(\alpha_{i}\right)\right\}$. By applying the Hölder inequality we obtain:

$$
\int_{E} f(x) d x \leq|E|^{\frac{1}{q}}\|f\|_{p},
$$

where $\frac{1}{p}+\frac{1}{q}=1$. Note that $|E|=\sum_{i=1}^{N}\left(\beta_{i}-\alpha_{i}\right)$. This, along with (3) and (4), will give the desired result, provided that $\delta<\left(\frac{\epsilon}{\|f\|_{p}}\right)^{q}$.

Corollary 3. The function $\xi$, as defined in (2), satisfies:

$$
\xi(s)=\int_{0}^{s} F(\tau) d \tau
$$

for some integrable function $F$.

Proof. By Theorem 2, $\xi$ is absolutely continuous. Hence we can apply Corollary 14 in [12], together with the fact that $\xi(0)=0$, to deduce

$$
\xi(s)=\int_{0}^{s} \xi^{\prime}(\tau) d \tau,
$$

almost everywhere in $[0,|D|]$. So by setting $F(s)=\xi^{\prime}(s)$, we get the desired result.

We now state our second main result:

Theorem 4. Let $F$ be the function in Corollary 3 and $\phi \in \mathcal{M}(D,[0,|D|])$. Then $F \circ \phi \in \overline{\mathcal{R}(f)}$.

Proof. Note that $\lambda_{F \circ \phi}(\alpha)=\lambda_{F}(\alpha)$, for every $\alpha \in \mathbb{R}$. Thus, $(F \circ \phi)^{*}(s)=F^{*}(s)$, for almost every $s \in[0,|D|]$. Hence, in view of item (3) of Lemma 1, it suffices to prove:

(i) $\int_{0}^{|D|} F^{*}(s) d s=\int_{0}^{|D|} f^{*}(s) d s$.

(ii) $\int_{0}^{s} F^{*}(t) d t \leq \int_{0}^{s} f^{*}(t) d t, \quad \forall s \in(0,|D|)$.

Proving (i) is straightforward as

$$
\begin{aligned}
& \int_{0}^{|D|} F^{*}(t) d t=\int_{0}^{|D|} F(t) d t=\xi(|D|) \\
&=\int_{\{u \geq t(|D|)\}} f d x=\int_{\{u \geq 0\}} f d x=\int_{D} f d x=\int_{0}^{|D|} f^{*}(t) d t,
\end{aligned}
$$

where we have used Corollary 3.

To prove (ii), we consider the following steps: 
Step 1 . Let $\mathcal{U}$ be an open subset of $(0,|D|)$. Then, we can write $\mathcal{U}=\cup_{i=1}^{\infty}\left(A_{i}, B_{i}\right)$, where $\left(A_{i}, B_{i}\right)$ are mutually disjoint. Hence,

$$
\begin{aligned}
\int_{\mathcal{U}} F(\tau) d \tau & =\sum_{i=1}^{\infty} \int_{A_{i}}^{B_{i}} F(\tau) d \tau=\sum_{i=1}^{\infty}\left(\int_{0}^{B_{i}} F(\tau) d \tau-\int_{0}^{A_{i}} F(\tau) d \tau\right) \\
& =\sum_{i=1}^{\infty}\left(\int_{\left\{u \geq t\left(B_{i}\right)\right\}} f d x-\int_{\left\{u \geq t\left(A_{i}\right)\right\}} f d x\right)=\sum_{i=1}^{\infty} \int_{\left\{t\left(B_{i}\right) \leq u<t\left(A_{i}\right)\right\}} f d x \\
& =\int_{\bigcup\left\{t\left(B_{i}\right) \leq u<t\left(A_{i}\right)\right\}} f d x \leq \int_{0}^{\left|\bigcup t\left(B_{i}\right) \leq u<t\left(A_{i}\right)\right|} f^{*}(s) d s \\
& =\int_{0}^{\sum\left(B_{i}-A_{i}\right)} f^{*}(s) d s=\int_{0}^{|\mathcal{U}|} f^{*}(s) d s .
\end{aligned}
$$

Step 2. Let $\mathcal{V}$ be a measurable subset of $(0,|D|)$, and $\epsilon>0$. By Theorem 3.6 in [15], there exists an open set $G$ containing $\mathcal{V}$ such that $|G \backslash \mathcal{V}|<\epsilon$. Whence

$$
\begin{aligned}
\int_{\mathcal{V}} F(t) d t \leq \int_{G} F(t) d t & \leq \int_{0}^{|G|} f^{*}(s) d s \\
& =\int_{0}^{|\mathcal{V}|} f^{*}(s) d s+\int_{|\mathcal{V}|}^{|G|} f^{*}(s) d s \\
& \leq \int_{0}^{|\mathcal{V}|} f^{*}(s) d s+\|f\|_{p}(|G|-|\mathcal{V}|)^{1 / q}
\end{aligned}
$$

where we have used Step 1, and Hölder's inequality. Since $|G|-|\mathcal{V}|=\mid G \backslash$ $V \mid<\epsilon$, from (6) we infer

$$
\int_{\mathcal{V}} F(t) d t \leq \int_{0}^{|\mathcal{V}|} f^{*}(s) d s+\epsilon^{1 / q}\|f\|_{p} .
$$

Since $\epsilon$ is arbitrary, (7) implies

$$
\int_{\mathcal{V}} F(t) d t \leq \int_{0}^{|\mathcal{V}|} f^{*}(s) d s
$$

Step 3. We recall the following maximization from [1]:

$$
\sup _{\{\omega \subseteq[0,|D|]:|\omega|=\gamma\}} \int_{\omega} F(t) d t=\int_{0}^{|\omega|} F^{*}(s) d s .
$$

Now, fix $s \in(0,|D|)$, and apply Step 3 to obtain

$$
\sup _{\{\omega \subseteq[0,|D|]:|\omega|=s\}} \int_{\omega} F(t) d t=\int_{0}^{s} F^{*}(t) d t .
$$


On the other hand, from Step 2, we have:

$$
\int_{\omega} F(t) d t \leq \int_{0}^{|\omega|} f^{*}(s) d s .
$$

From (8) and (9) we deduce

$$
\int_{0}^{s} F^{*}(t) d t \leq \int_{0}^{s} f^{*}(t) d t
$$

as desired.

Corollary 5. Suppose the hypotheses of Theorem 4 hold. Then, there exists a sequence of functions $\left\{F_{n}\right\}$ such that $F_{n}^{*}(s)=f^{*}(s)$ and $F_{n} \rightarrow F$ in $L^{p}(0,|D|)$.

Proof. By Ryff's result, $f=f^{*} \circ \phi$, for some $\phi \in \mathcal{M}(D,[0,|D|])$. From Theorem 4 , we infer $F \circ \phi \in \overline{\mathcal{R}(f)}$. So, there exists a sequence $\left\{f_{n}\right\} \subseteq \mathcal{R}(f)$ such that $f_{n} \rightarrow F \circ \phi$ in $L^{p}(D)$. Therefore, $f_{n} \circ \phi^{-1} \rightarrow F$ in $L^{p}(0,|D|)$. Clearly, $\lambda_{f_{n} \circ \phi^{-1}}(\alpha)=\lambda_{f}(\alpha)$, so $\left(f_{n} \circ \phi^{-1}\right)^{*}(s)=f^{*}(s)$. This completes the proof.

\section{Applications}

In this section we will present a couple of applications of the results of the previous section. Throughout we will assume the extra condition $f \in C(\bar{D})$. Let us consider the following Hamilton-Jacobi system:

$$
\begin{cases}|\nabla u|=f(x), & \text { in } D \\ u=0 & \text { on } \partial D .\end{cases}
$$

Lemma 6. The system (10) has a strong positive solution $u \in W^{1, \infty}(D)$.

Proof. From [10] we know that the system (10) has a strong solution $u \in W^{1, \infty}(D)$. Replacing $u$ by $|u| \in W^{1, \infty}(D)$ if necessary, taking into account that $|\nabla(|u|)|=|\nabla u|$, we can assume $u$ is non-negative. On the other hand, since $f$ is positive, we can apply Lemma 7.7 in [7], to ensure that the level sets $\{u=\gamma\}$ have zero measure. Thus, $u$ is essentially positive, as desired.

Remark 1. For $f$ and $u$ as in Lemma 6, the function:

$$
\xi(s)=\int_{\{u \geq t\}} f(x) d x, \quad\left(\text { where } s=\lambda_{u}(t)\right),
$$

is well defined. As a result, the function $F$ from Corollary 3 is also well defined. Moreover, the conclusions of Theorem 2 and Theorem 4 hold. 
Our first application is as follows:

Theorem 7. Let $u \in W^{1, \infty}(D)$ be a strong positive solution of the Hamilton-Jacobi system (10) and let $v$ be the unique solution of the following system:

$$
\begin{cases}|\nabla Z|=F\left(\omega_{n}|x|^{n}\right), & \text { in } B \\ Z=0, & \text { on } \partial B,\end{cases}
$$

in which:

- $B$ is the ball centred at the origin with radius $\left(|D| / \omega_{n}\right)^{1 / n}$, and $\omega_{n}$ indicates the volume of the unit n-dimensional ball.

- The function $F$ is as in Corollary 3, which is well defined by Remark 1.

Also, let $u^{\sharp}(x) \equiv u^{*}\left(\omega_{n}|x|^{n}\right)$, which in the literature is referred to as the Schwarz symmetrization of $u$. Then, $u^{\sharp}(x) \leq v(x)$, for $x \in B$.

Proof. The proof is a consequence of Corollary 3, along the same lines as in the proof of Lemma 2.2 in [6].

Example 1. Choosing $f(x)=1$ in Theorem 7 yields $F(t)=1$. Thus, the conclusion of Theorem 7 states:

$$
u^{\sharp}(x) \leq v(x)=R-|x|, \quad x \in B,
$$

where $R=\left(|D| / \omega_{n}\right)^{1 / n}$. This estimate can be obtained directly as follows:

$$
\begin{aligned}
\lambda_{u}(t)=\int_{\{u \geq t\}} d x & =\int_{\{u \geq t\}}|\nabla u| d x \\
& =\int_{t}^{\|u\|_{\infty}}\left(\int_{\{u=\tau\}} d H^{n-1}\right) d \tau=\int_{t}^{\|u\|_{\infty}} P(\{u \geq \tau\}) d \tau,
\end{aligned}
$$

where we have used the co-area formula (e.g. see [11]). Here, $P(E)$ stands for the perimeter of $E$ in the sense of De Giorgi. By differentiating (12), and applying the classical Isoperimetric Inequality (e.g. see [8]), we derive:

$$
\lambda_{u}^{\prime}(t)=-P(\{u \geq t\}) \leq-n \omega_{n}^{\frac{1}{n}} \lambda_{u}^{1-\frac{1}{n}}(t) .
$$

Thus, we obtain:

$$
1 \leq-\frac{\lambda_{u}^{\prime}(t)}{n \omega_{n}^{\frac{1}{n}} \lambda_{u}^{1-\frac{1}{n}}(t)} .
$$


Integrating (13) from 0 to $t$ leads to:

$$
\begin{aligned}
t \leq-\frac{1}{n \omega_{n}^{1 / n}} \int_{0}^{t} \frac{\lambda_{u}^{\prime}(\tau)}{\lambda_{u}^{1-\frac{1}{n}}(\tau)} d \tau & =-\frac{1}{n \omega_{n}^{1 / n}} \int_{|D|}^{\lambda_{u}(t)} \frac{d s}{s^{1-\frac{1}{n}}} \\
& =\frac{1}{\omega_{n}^{1 / n}}\left(|D|^{1 / n}-\lambda_{u}^{1 / n}(t)\right)=R-\left(\frac{\lambda_{u}(t)}{\omega_{n}}\right)^{1 / n} .
\end{aligned}
$$

By letting $t=u^{*}\left(\omega_{n}|x|^{n}\right)$ in (14), and recalling $\lambda_{u}\left(u^{*}\left(\omega_{n}|x|^{n}\right)\right)=\omega_{n}|x|^{n}$, we obtain $u^{\sharp}(x) \leq R-|x|$ for $x \in B$, as expected.

The second application is stated in the following Theorem:

Theorem 8. Let $u$ be as in Theorem 7. Then

$$
\|u\|_{\infty} \leq C|D|^{1 / n}\|f\|_{\infty} .
$$

Proof. The proof is a consequence of Corollary 5, along the same lines as in the proof of Corollary 2.1 in [6].

Acknowledgment. The authors wish to thank the referees for their constructive critique of the first draft.

\section{References}

[1] A. Alvino, G. Trombetti, and P. L. Lions. On optimization problems with prescribed rearrangements. Nonlinear Anal., 13(2):185-220, 1989.

[2] M. Belloni, V. Ferone, and B. Kawohl. Isoperimetric inequalities, Wulff shape and related questions for strongly nonlinear elliptic operators. Z. Angew. Math. Phys., 54(5):771-783, 2003.

[3] G. R. Burton. Rearrangements of functions, maximization of convex functionals, and vortex rings. Math. Ann., 276(2):225-253, 1987.

[4] G. R. Burton. Variational problems on classes of rearrangements and multiple configurations for steady vortices. Ann. Inst. H. Poincaré Anal. Non Linéaire, 6(4):295-319, 1989.

[5] A. Eydeland and B. Turkington. On the computation of nonlinear planetary waves. Stud. Appl. Math., 76(1):37-67, 1987.

[6] E. Giarrusso and D. Nunziante. Symmetrization in a class of first-order Hamilton-Jacobi equations. Nonlinear Anal., 8(4):289-299, 1984. 
[7] D. Gilbarg and N. S. Trudinger. Elliptic Partial Differential Equations of Second Order. Springer, Berlin, 2001.

[8] A. Henrot. Extremum Problems for Eigenvalues of Elliptic Operators. Birkhäuser, Basel, 2006.

[9] B. Kawohl. Rearrangements and Convexity of Level Sets in PDE. Number 1150 in Lecture Notes in Math. Springer, Berlin, 1985.

[10] P.-L. Lions. Generalized Solutions of Hamilton-Jacobi Equations. Number 69 in Res. Notes Math. Pitman, 1982.

[11] F. Morgan. Geometric Measure Theory: A Beginner's Guide. Elsevier/Academic Press, Burlington, fourth edition, 2009.

[12] H. L. Royden. Real Analysis. The Macmillan Co., New York; CollierMacmillan Ltd., London, 1963.

[13] J.V. Ryff. Measure preserving transformations and rearrangements. J. Math. Anal. Appl., 31:449-458, 1970.

[14] G. Talenti. Elliptic equations and rearrangements. Ann. Sc. Norm. Super. Pisa Cl. Sci. 4e série, 3(4):697-718, 1976.

[15] R. L. Wheeden and A. Zygmund. Measure and Integral. An Introduction to Real Analysis, volume 43 of Pure and Applied Mathematics. Marcel Dekker, Inc., New York-Basel, 1977. 